\title{
Quantifying secular evolution through structural decomposition
}

\author{
Lee Kelvin ${ }^{1,2,3}$ \\ ${ }^{1}$ School of Physics \& Astronomy, University of St Andrews, St Andrews, KY16 9SS, UK \\ ${ }^{2}$ ICRAR, The University of Western Australia, 35 Stirling Hwy, WA 6009, Australia \\ ${ }^{3}$ Inst für Astro- u Teilchenphysik, Universität Innsbruck, Techstr 25, 6020 Innsbruck, Austria \\ email: lee.kelvin@uibk.ac.at
}

\begin{abstract}
Structure within a galaxy is not random, instead emerging as a direct function of its evolutionary path. It is thought that secular evolutionary processes leave behind distinct structural tracers in the form of bars, pseudo-bulges and rings. We have developed a robust automated structural analysis pipeline (Kelvin et al., 2012) able to accurately map structure across a range of ground and space-based datasets. Using reprocessed SDSS and UKIDSS data from the GAMA survey: an imaging and spectroscopic survey with over 300, 000 redshifts across 300 square degrees (Driver et al., 2009); we measure the relative abundance and stellar mass locked up within these structures in the local $(z<0.06)$ Universe. Future robust calculations of the stellar mass budget within bulges, bars, disks and pseudo-bulges should allow us to measure the relative importance of secular evolution against other mechanisms across cosmic time.
\end{abstract}

Keywords. galaxies: evolution, galaxies: structure, techniques: image processing, astronomical data bases: miscellaneous, galaxies: bulges, galaxies: spiral, galaxies: fundamental parameters

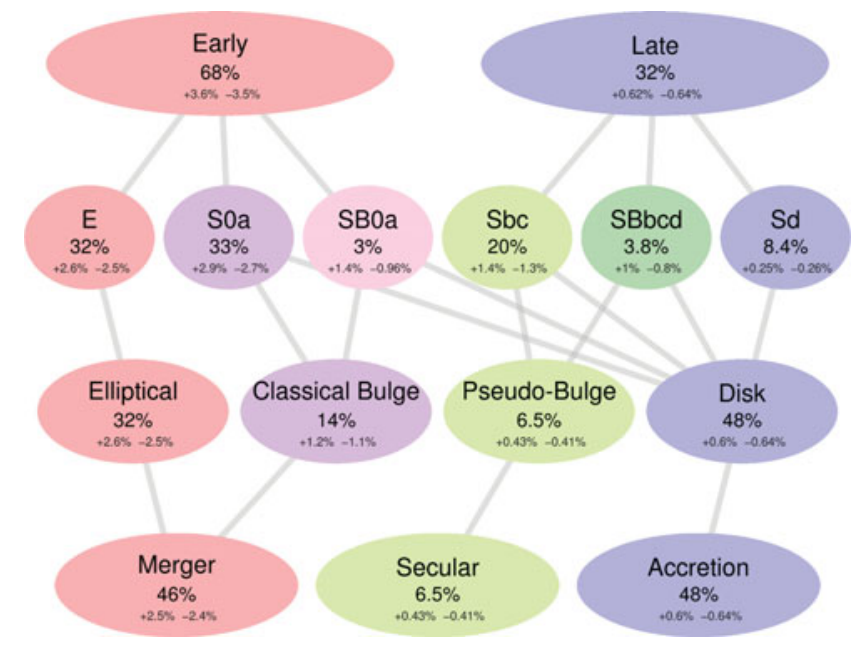

Figure 1. The breakdown of galaxy stellar mass in the local Universe by (top to bottom): morphological type; morphological class; galaxy structure, and; evolutionary processes. Percentages represent the fraction of mass within that division, with $2 \sigma$ errors shown below for reference.

\section{References}

Driver. S. P. et al., 2009, Astron. Geophys. 50, 050000

Kelvin, L. S. et al., 2012, MNRAS 421, 1007 